\title{
A conservative assessment of the current constraints on dark matter annihilation from Cosmic Rays and CMB observations
}

\author{
Nicolò Masi ${ }^{\mathrm{a}, \mathrm{b}, *}$, Mario Ballardini $\mathrm{a}^{\mathrm{a}, \mathrm{c}, \mathrm{b}}$ \\ ${ }^{a}$ DIFA, Dipartimento di Fisica e Astronomia, Alma Mater Studiorum Università di Bologna, \\ viale Berti Pichat 6/2, I-40127 Bologna, Italy \\ ${ }^{b}$ INFN, Sezione di Bologna, \\ via Irnerio 46, I-40126 Bologna, Italy \\ ${ }^{c} I N A F / I A S F-B O$, Istituto di Astrofisica Spaziale e Fisica Cosmica di Bologna, \\ via Gobetti 101, I-40129 Bologna - Italy
}

\section{Abstract}

OD In view of the current interest in combining different observations to constraint annihilating WIMP dark matter, we examine the relation between the Sommerfeld effect at the recombination epoch and in the galactic halo. By considering an up-to-date collection of interpolations of cosmic rays lepton data (AMS-02 2014, Fermi and PAMELA), as dark matter annihilation signals, we show that current cosmic rays measurements and recent Planck 2015 constraints from CMB anisotropies almost overlap for dark matter masses of the order of few $T e V$, although great theoretical uncertainties afflict cosmic rays and dark matter descriptions. Combining cosmic rays fits we obtain proper minimal regions allowed by CMB observations, especially for $\mu$ and $\tau$ annihilation channels, once assumed viable values of the efficiency factor for energy absorption at recombination: the results are consistent with those obtained by the Planck collaboration but allow a slightly larger overlap between Cosmic Rays constraints from the lepton sector and CMB. Incoming AMS-02 measurements of cosmic rays antiprotons will help to clarify the conundrum.

Keywords: Dark matter, AMS-02, Planck

PACS:

\section{Introduction}

AMS-02 2014 measurements of cosmic rays (CR) leptons [1, 2, 3, which have confirmed the rise of the positron fraction for kinetic energy above $\sim 10 \mathrm{GeV}$, up to $\sim 1 \mathrm{TeV}$, have stimulated different interpretations of this excess of positrons as primary evidence of dark matter (DM) annihilation. The interpretation of DM as a new source of positrons, to explain the departure from the pure secondary positron fraction, can be tested with other complementary and independent indirect measurements, as the cosmic microwave background (CMB) anisotropies. In fact, if DM particles self-annihilate at a sufficient rate, the expected signal would be directly sensitive to their thermally averaged cross section and it could have drawn an imprint on CMB temperature and polarization anisotropies.

The aim of the present letter is to discuss the link between the DM annihilation cross sections at freeze-out, recombination and in the Milky Way galactic halo, which is essential to compare different indirect constraints on DM itself. The three different physical quantities are defined

\footnotetext{
*Corresponding author

Email addresses: masi@bo.infn.it (Nicolò Masi), ballardini@iasfbo.inaf.it (Mario Ballardini)
}

by the environment in three different epochs. The relation between them is not trivial, not a simply decreasing function and so it must be carefully analyzed to put coherent constraints on DM properties. Once defined a consistent framework, one can compare information from $\mathrm{CR}$ physics with CMB observations.

The need of a high thermally averaged annihilation cross section, not purely thermal, i.e. $\langle\sigma \nu\rangle^{\text {ann }} \gg 3 \times$ $10^{-26} \mathrm{~cm}^{3} \mathrm{~s}^{-1}$, comes from the above mentioned observation of a huge excess in the $\mathrm{CR}$ positron fraction, in contrast with the expected behavior of secondaries produced in the interstellar medium (ISM). In order to describe this experimental evidence, a very high annihilation cross section has to be invoked, compared with typical expectation for a s-wave annihilating thermal relic matching the observed DM abundance [4.

The main way to interpret the positron excess is the Sommerfeld enhancement [5], a non-perturbative quantum effect which modifies the annihilation cross section in the regime of small relative velocity of the annihilating particles and in presence of an effectively long-range force between them. Indeed, this well-known quantum mechanical effect can occur in DM annihilations in the galactic halo, if the two annihilating particles exchange an interaction mediated by a force carrier. From a Feynman diagram point of view, the Weakly Interacting Massive Particles (WIMP) 
interact with the new boson through a multi-box diagram, depicted in Fig. 1, annihilate into $\chi \chi \rightarrow \phi \phi$, and the decay of $\phi$ produces more light leptons than expected.

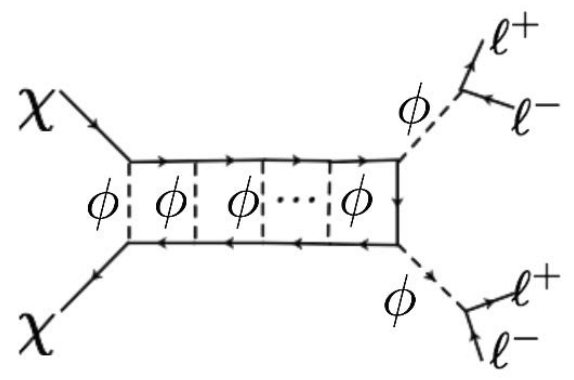

Figure 1: Feynman diagram for the Sommerfeld enhancement induced by a scalar.

The structure of the paper is the following: in Section 2 the basic setup of the Sommerfeld enhancement and the relation between the galactic halo cross section and the one at recombination are briefly reviewed; in Section 3 we report a collection of $\mathrm{CR}$ best fits for $\mathrm{TeV}$-ish $\mathrm{DM}$ candidates capable to reproduce the observed positron excess and we discuss the main sources of uncertainties (from CR physics and the DM sector), in order to compare predictions with $\mathrm{CMB}$ constraints and obtain some general remarks in Section 4

\section{The quantum Sommerfeld enhancement}

The Sommerfeld enhancement is fundamental to interpret indirect DM searches. The thermally averaged DM annihilation cross section (at any time) can be generally decomponed into powers of the velocity $\nu[5]$ :

$$
\begin{aligned}
\langle\sigma \nu\rangle^{\mathrm{ann}} & =\sum_{n=0}^{\infty} c_{n}\left\langle\nu^{2 n}\right\rangle+\text { Sommerfeld effect } \\
& \sim A+B\left\langle\nu^{2}\right\rangle+C(\bar{\nu}+\langle\nu\rangle)^{-1}+\mathcal{O}\left(\left\langle\nu^{4}\right\rangle\right)
\end{aligned}
$$

where $\nu$ is the relative velocity of the annihilating particles, that is $\nu=\nu_{\text {rel }}=2 \nu_{\mathrm{CM}}, A$ is the constant s-wave term, $B \nu^{2}$ is the p-wave term and $C(\bar{\nu}+\langle\nu\rangle)^{-1}$ the Sommerfeld term where $\bar{\nu}$ stands for a proper asymptotic (cut-off) value for the low velocity regime. The quadratic and quartic terms in Eq. (1) are commonly neglected at a freeze-out description, whereas the so called Sommerfeld term is suppressed in the relativistic velocities regime [6].

The enhancement of the s-wave for these velocities is defined by the ratio of the masses of the DM candidate and the Sommerfeld boson $\phi$ which provides the boosted annihilation, and by the effective coupling $g_{\chi} \approx \sqrt{4 \pi \alpha_{\chi}}$. Defining the dimensionless parameter $\varepsilon_{\phi}^{-1}=\alpha_{\chi} m_{\mathrm{DM}} / m_{\phi}$, the condition for the enhancement is $\varepsilon_{\phi}^{-1}>1$, that is $m_{\phi} / m_{\mathrm{DM}} \lesssim 10^{-(3 \div 2)}$ and $\alpha_{\chi} \gtrsim 10^{-(2 \div 1)}$.

In Fig. 2 the different contributions to the total DM annihilation cross section are shown according to Eq. (1). It

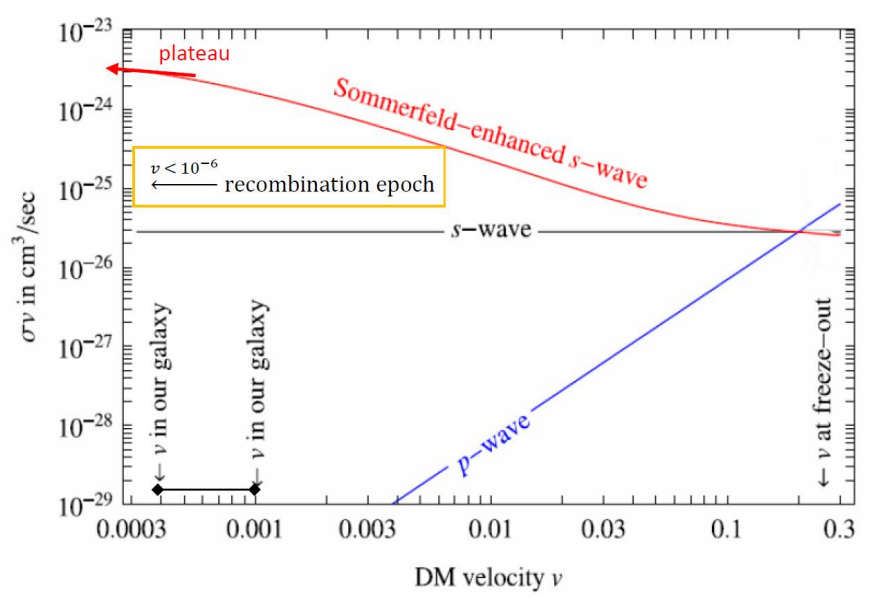

Figure 2: The three main terms of the DM annihilation cross section as functions of $\beta$ ( $\nu$ in natural unit on the abscissa). See 6] for insights.

can be noted that the Sommerfeld contribution is a function of the redshift $z$ but, after a particular value of $\nu$ $\left(\sim 10^{-4} c\right)$, which is very close to the one expected for our dark halo, the quantum effect reaches a plateau that is approximately 100 times the thermal annihilation cross section, i.e. $3 \times 10^{-24} \mathrm{~cm}^{3} \mathrm{~s}^{-1}$. Here one can notice that there should be necessarily a slow velocity limit, given by the finite range of the attractive force mediated by $\phi$ : once the de Broglie wavelength of the particle $\left(m_{\mathrm{DM}} \nu\right)^{-1}$ exceeds the range of the interaction $m_{\phi}^{-1}$, the quantum effect saturates reaching the value $S_{\max } \sim \alpha_{\chi} m_{\mathrm{DM}} / m_{\phi}$, that is a constant and does not depend on $\nu$ 7]. Moreover, for specific values of $\varepsilon_{\phi}$ and $\varepsilon_{\nu} \equiv \beta / \alpha_{\chi}$, where $\beta$ is the ratio of $\nu$ to the speed of light $c$, resonant threshold states can be produced (see Fig. 3) which are capable of further boosting $\langle\sigma \nu\rangle$, , inducing $S \geq 10^{3}$.

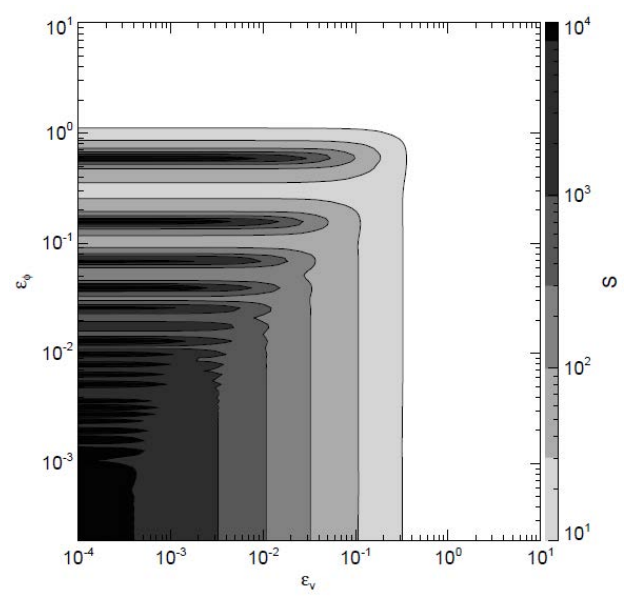

Figure 3: Sommerfeld boost in the $\varepsilon_{\phi}-\varepsilon_{\nu}$ plane [8].

After cosmological recombination, DM had a velocity proportional to the inverse of the scale factor of the expanding Universe because of its free stream, justifying very small velocity values; on the other hand, the virialized ve- 
locities in a today galactic halo might be at least three order of magnitude greater.

The status of the three cross sections in the previously mentioned regimes can be basically summarized as follows:

1. Freeze-out. During this phase the cross section is usually assumed to be nearly constant, without the general contribution of other even powers of $\nu$. This is an absolute minimum from which the annihilation cross section evolves during the cooling of the Universe. As shown in Fig. 2, for "too relativistic" freeze-out velocities, the pwave term would enhance the annihilation cross section of one order of magnitude, generating an incompatibility w.r.t. the expected relic density at redshift $z=0$. For this regime the relative velocity is:

$$
\left.\nu\right|_{z \gg 1000}=\nu_{\text {freeze-out }} \lesssim 0.3 c
$$

and the following relation for the cross section holds:

$$
\langle\sigma \nu\rangle_{\text {therm }}^{\text {ann }} \sim 3 \times 10^{-26} \mathrm{~cm}^{3} \mathrm{~s}^{-1} .
$$

2. Recombination. Here the annihilation cross section has a greater value than the freeze-out one, i.e. $\left.\langle\sigma \nu\rangle_{\text {rec }}\right\rangle$ $\langle\sigma \nu\rangle_{\text {therm }}$. This does not imply the relation $\left.\langle\sigma \nu\rangle_{\text {rec }}\right\rangle$ $\langle\sigma \nu\rangle_{\text {halo }}$, because the quantum Sommerfeld enhancement saturates after a certain velocity $\nu_{\text {sat }}$, which is $\nu_{\text {sat }} \lesssim 10^{-3} c$. So, for $\nu<\nu_{\text {sat }}$ the quantum Sommerfeld boost $S(z)$ does not increase the annihilation cross section further. This is the annihilation cross section measured by CMB experiments. In this regime the relative velocity of the DM particles is very low:

$$
\left.\nu\right|_{600<z<1000}=\nu_{\mathrm{rec}} \sim 10^{-(8 \div 6)} c
$$

and the annihilation cross section is clearly dominated by the Sommerfeld enhancement:

$$
\langle\sigma \nu\rangle_{\mathrm{rec}}^{\mathrm{ann}}=S_{\mathrm{rec}}\langle\sigma \nu\rangle_{\mathrm{therm}} .
$$

3. Galactic halo. As for recombination epoch, the dominant term is the Sommerfeld one. In our Galaxy the velocity of the DM particles is supposed to be of the order of $(100 \div 300) \mathrm{km} \mathrm{s}^{-1}$, which implies $\nu \lesssim 10^{-3} \mathrm{c}$. In the Galaxy the velocity regime is:

$$
\left.\nu\right|_{z=0}=\nu_{\text {halo }} \sim 5 \times 10^{-4} c
$$

with an annihilating cross section:

$$
\langle\sigma \nu\rangle_{\text {halo }}^{\text {ann }}=S_{\text {halo }}\langle\sigma \nu\rangle_{\text {therm }} .
$$

If we take into account that the current value of $\nu$ in our Galaxy does not completely saturate the Sommerfeld effect, a conservative numerical factor 2 can be assumed for the ratio $S_{r e c} / S_{\text {halo }}$ between the enhancement at recombination and the one today (see Fig. 2). The relation between the galactic annihilation cross section measured by AMS-02 and the one measured by Planck at the recombination can be written as:

$$
\langle\sigma \nu\rangle_{\mathrm{rec}}^{\mathrm{ann}} \approx(1 \div 2)\langle\sigma \nu\rangle_{\mathrm{halo}}^{\mathrm{ann}}
$$

\section{Constraints on dark matter properties from as- troparticle physics and cosmology}

The CMB experiments can constrain the DM annihilation cross section from the quantity introduced in Refs. [9, 10]:

$$
p_{\text {ann }} \equiv f_{\text {eff }} \frac{\langle\sigma \nu\rangle_{\text {rec }}}{m_{\mathrm{DM}}}
$$

as a function of the factor $f_{\text {eff }}$ that encodes the efficiency of the energy absorption at the recombination. Therfore, another parameter has to be taken into account for the comparison between $\mathrm{CR}$ and $\mathrm{CMB}$ experiments. In principle $f_{\text {eff }}=f(z)$, but it has been demonstrated that this can be taken as a constant at $z \simeq 600$ [11, 12, around the recombination epoch; it lies in the theoretical range between 0.01 and 1 , with recombination values generally chosen between 0.12 and 0.6 as a function of the annihilation channel (see Refs. 8, 13, 12] ). So, for small $f_{\text {eff values }}$ the annihilation constraints are relaxed.

In Refs. [13, 7] have been obtained the following bounds for the saturated annihilation cross section and the Sommerfeld factor from WMAP 5 yr data:

$$
\begin{aligned}
\langle\sigma \nu\rangle_{\mathrm{sat}} & <\frac{3 \times 10^{-24} \mathrm{~cm}^{3} \mathrm{~s}^{-1}}{f_{\mathrm{eff}}}\left(\frac{m_{\mathrm{DM}}}{1 T e V}\right) \\
S_{\mathrm{max}} & <\frac{120}{f_{\mathrm{eff}}}\left(\frac{m_{\mathrm{DM}}}{1 T e V}\right) .
\end{aligned}
$$

For $1 \mathrm{TeV}$ DM candidate and $f_{\text {eff }}=0.12$ Eqs. $10-11$ lead to $\langle\sigma \nu\rangle_{\text {sat }}<2.5 \times 10^{-23} \mathrm{~cm}^{3} \mathrm{~s}^{-1}$ and $S_{\max }<10^{3}$. Now, with the latest Planck 2015 data 14 this constraint can be improved of about one order of magnitude (see in Fig. 4 the comparison between WMAP9 and Planck constraints), leading, for the previous case, to $\langle\sigma \nu\rangle_{\text {sat }}<$ $10^{-24} \mathrm{~cm}^{3} \mathrm{~s}^{-1}$ and $S_{\max }<10^{2}$.

In Fig. 4 the constraints from Planck [14, obtained with the full temperature data and the inclusion of lowand high- $l$ polarization data, are shown. Here, the allowed region of parameters space for charged CR measurements was taken from Cholis and Hooper [15], under the assumption that the CR positron excess was due to pure DM annihilation.

From Fig. 4 it can be noted that, for a TeV-ish particle, e.g. with a mass of $3 \mathrm{TeV}$, and a pessimistic case with an ideal absorption efficiency $f_{\text {eff }}=1$, the annihilation cross section at recombination from Planck is $\langle\sigma \nu\rangle_{\text {rec }}\left\langle 10^{-24} \mathrm{~cm}^{3} \mathrm{~s}^{-1}\right.$, which implies $\langle\sigma \nu\rangle_{\text {halo }}<0.5 \times 10^{-24} \mathrm{~cm}^{3} \mathrm{~s}^{-1}$ at most. Instead, in an optimistic case with $\langle\sigma \nu\rangle_{\mathrm{rec}} \approx\langle\sigma \nu\rangle_{\text {halo }}$ and $f_{\text {eff }}=0.1$, the constraint for a TeV-ish DM becomes $\langle\sigma \nu\rangle<10^{-23} \mathrm{~cm}^{3} \mathrm{~s}^{-1}$.

Several studies suggest values less than 0.4 for $f_{\text {eff }}$ at recombination, that is a rather optimistic scenario for indirect search constraints [16, 17]. Consequently, constraints of the order of $10^{-(24 \div 23)} \mathrm{cm}^{3} \mathrm{~s}^{-1}$ for a heavy DM candidate can be still in agreement with the boosted cross

\footnotetext{
${ }^{2}$ We thank Silvia Galli for clarifying this point.
} 


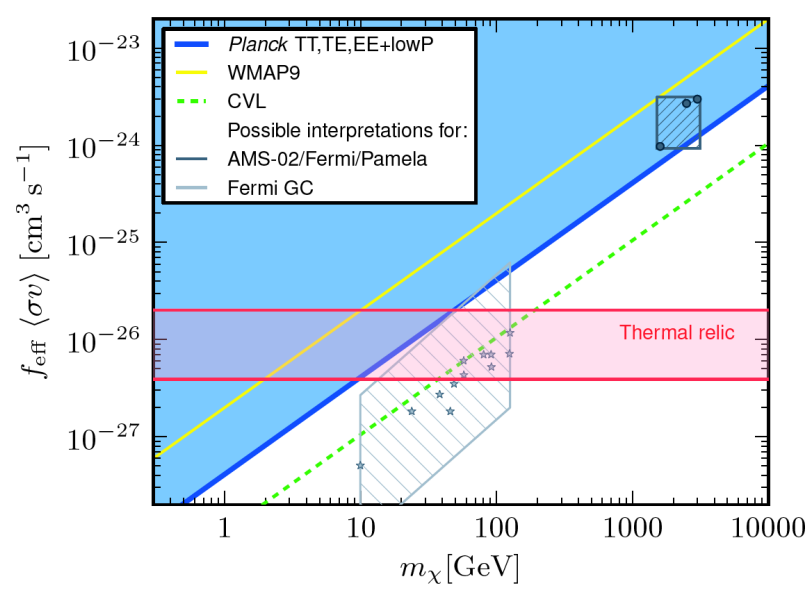

Figure 4: Annihilation cross section constraint as a function of DM mass. (Image credit: ESA and the Planck Collaboration, 14.) The AMS-02/Fermi/PAMELA rectangle was obtained from Ref. 15] considering $\left(m_{\mathrm{DM}}=1.6 \mathrm{TeV},\langle\sigma \nu\rangle=6.5 \times 10^{-24} \mathrm{~cm}^{3} \mathrm{~s}^{-1}, f_{\mathrm{eff}}=0.15\right)$, $\left(m_{\mathrm{DM}}=2.5 \mathrm{TeV},\langle\sigma \nu\rangle=1.5 \times 10^{-23} \mathrm{~cm}^{3} \mathrm{~s}^{-1}, f_{\mathrm{eff}}=0.18\right)$ and $\left(m_{\mathrm{DM}}=3 \mathrm{TeV},\langle\sigma \nu\rangle=2.3 \times 10^{-23} \mathrm{~cm}^{3} \mathrm{~s}^{-1}, f_{\mathrm{eff}}=0.13\right)$ for $2 e / 2 \mu / 2 \pi, 4 \mu$ and $4 \pi$ respectively ${ }^{2}$

sections which are necessary to reproduce CR positrons excess [5]; as a first step, only resonant Sommerfeld boosts, that induce an overall enhancement $>10^{3}$ w.r.t $\langle\sigma \nu\rangle_{\text {therm }}$, can be for certain excluded.

It follows that it is interesting and necessary to discuss and update the small rectangle in the right upper corner of Fig. 4, related to AMS-02 2013 [18, Fermi [19] and PAMELA 20] data, in light of new CR results and speculations, to avoid falling into misleading conclusions.

In fact, the analysis in [15] was performed with only 2013 AMS-02 data along with PAMELA, Fermi and AMS-01 ones. It must be also noted that Fermi all electrons channel $e^{+}+e^{-}$is in tension with AMS-02 positron fraction, as stressed in [21], and it has not been reprocessed and confirmed after the recent pass 8 calibration performed in $22] 3$

Furthermore, putting all these CR data together could produce some inconsistencies and issues: since the data used for fits were collected by different instruments, the fits errors are hard to estimate.

The most interesting constraints in literature for TeVish DM candidates, i.e. $m_{\mathrm{DM}} \geq 1 \mathrm{TeV}$, using AMS-02 positron data are the following:

1. From the previously discussed Ref. [15] it must be noted that the reduced $\tilde{\chi}^{2}$ for $\mathrm{TeV}$-ish $\mathrm{DM}$ fits is much better for Fermi data rather than for AMS-02 2013 ones. The good fits, with $\tilde{\chi}^{2} \sim 1$, for AMS-02 data in [18, for a DM which annihilates into a pair of intermediate states $\phi$ are $\langle\sigma \nu\rangle=5.8 \times 10^{-24} \mathrm{~cm}^{3} \mathrm{~s}^{-1}$ with $m_{\mathrm{DM}}=1 \mathrm{TeV}$,

\footnotetext{
${ }^{3}$ Peter Michelson, Fermi-LAT PI, confirms the non reliability of Fermi $e^{+}+e^{-}$data (and consequently of positron fraction and $e^{+}$data) at the AMS-days at CERN on April 2015, see https://cds.cern.ch/record/2010841 at $18^{\prime}$.
}

$\langle\sigma \nu\rangle=6.5 \times 10^{-24} \mathrm{~cm}^{3} \mathrm{~s}^{-1}$ with $m_{\mathrm{DM}}=1.6 \mathrm{TeV}$, $\langle\sigma \nu\rangle=1.5 \times 10^{-23} \mathrm{~cm}^{3} \mathrm{~s}^{-1}$ with $m_{\mathrm{DM}}=2.5 \mathrm{TeV}$ and $\langle\sigma \nu\rangle=2.3 \times 10^{-23} \mathrm{~cm}^{3} \mathrm{~s}^{-1}$ with $m_{\mathrm{DM}}=3 \mathrm{TeV}$ for $4 \mu, 4 \pi$ and $2 e / 2 \pi / 2 \mu$ combinations. The overall result is a DM with a mass up to $3 \mathrm{TeV}$ and an annihilation cross section in the range of few $10^{-(24 \div 23)} \mathrm{cm}^{3} \mathrm{~s}^{-1}$;

2. In Ref. 21] some fits to AMS-02 2014 positron fraction data with $\mathrm{TeV}$-ish $\mathrm{DM}$ are obtained: $\langle\sigma \nu\rangle \approx$ $4.5 \times 10^{-23} \mathrm{~cm}^{3} \mathrm{~s}^{-1}$ with $m_{\mathrm{DM}} \approx 1.76 \mathrm{TeV}$ for $4 \tau$ channel, $\langle\sigma \nu\rangle \approx(2.5 \div 7) \times 10^{-23} \mathrm{~cm}^{3} \mathrm{~s}^{-1}$ with $m_{\mathrm{DM}} \approx$ $(1 \div 2) \mathrm{TeV}$, with little deviations as a function of the annihilation channels combinations. Some fits are achieved using also Fermi-LAT data, which have a great uncertainty w.r.t. AMS-02 2014 data.

3. In Ref. 6] the privileged regions, using AMS-02 2013 data, are about $m_{\mathrm{DM}} \approx 1 \mathrm{TeV}$ with $\langle\sigma \nu\rangle \approx$ $10^{-23} \mathrm{~cm}^{3} \mathrm{~s}^{-1}$. They also used Fermi and HESS data;

4. In [23], using AMS-02 2013 data, along with PAMELA and Fermi ones, they obtain $m_{\mathrm{DM}} \approx 1 \mathrm{TeV}$ with $\langle\sigma \nu\rangle \approx$ $6 \times 10^{-24} \mathrm{~cm}^{3} \mathrm{~s}^{-1}$;

5. In Ref. [24, using AMS-02 2013, the results for a Sommerfeld boost $S$ from a scalar, pseudoscalar or vector particle are approximately the same: $m_{\mathrm{DM}} \approx 1 \mathrm{TeV}$ with $S \approx 1.5 \times 10^{2}$ and $\langle\sigma \nu\rangle=S\langle\sigma \nu\rangle_{\text {therm }} \approx 4.5 \times$ $10^{-24} \mathrm{~cm}^{3} \mathrm{~s}^{-1}$ for the $4 \mu$ channel, $m_{\mathrm{DM}} \approx(1.5 \div 2) \mathrm{TeV}$ with $S \approx(5 \div 9) \times 10^{2}$ and $\langle\sigma \nu\rangle=S\langle\sigma \nu\rangle_{\text {therm }} \approx$ $(1.5 \div 2.7) \times 10^{-23} \mathrm{~cm}^{3} \mathrm{~s}^{-1}$ for the $2 \tau$ channel, $m_{\mathrm{DM}} \approx$ $(3 \div 4) \mathrm{TeV}$ with $S \approx(1 \div 2) \times 10^{3}$ and $\langle\sigma \nu\rangle=$ $S\langle\sigma \nu\rangle_{\text {therm }} \approx(3 \div 6) \times 10^{-23} \mathrm{~cm}^{3} \mathrm{~s}^{-1}$ for the $4 \tau$ channel;

6. In Ref. [25, from AMS-02 2013 positron fraction data the upper limit of $\langle\sigma \nu\rangle \approx 10^{-(24 \div 23)} \mathrm{cm}^{3} \mathrm{~s}^{-1}$ is achieved, as a function of the annihilation channels, associated to a $m_{\mathrm{DM}}=1 \mathrm{TeV}$. They also use AMS-02 positrons from ICRC 2013, which were not finalized nor official data;

7. In Ref. [26], the best-fits obtained from AMS-02 2014 data are about $m_{\mathrm{DM}}=1(4) \mathrm{TeV}$ with $\langle\sigma \nu\rangle \approx 0.5(7) \times$ $10^{-23} \mathrm{~cm}^{3} \mathrm{~s}^{-1}$, for $\mu$ and $\tau$ channels;

8. In Ref. [27], the AMS-02 2014 data lead to a best-fit via Sommerfeld boson that is of the order of $m_{\mathrm{DM}}=$ $(1 \div 2) \mathrm{TeV}$ with $\langle\sigma \nu\rangle \approx(1 \div 5) \times 10^{-23} \mathrm{~cm}^{3} \mathrm{~s}^{-1}$, for $4 \mu$ and $\tau$ channels.

These studies share common properties and weaknesses. First of all the allowed and privileged leptonic annihilation channels for DM with mass $1 \mathrm{TeV} \leq m_{\mathrm{DM}}<10 \mathrm{TeV}$ are generally the muonic and tauonic ones. Also annihilation into quarks $u, d, b$ [21] and $\pi$ [15] are suitable for heavy DM. These best-fits are usually computed with DM masses $m_{\mathrm{DM}} \gtrsim 1 \mathrm{TeV}$, however mass values in the $(4 \div 10) \mathrm{TeV}$ range, the most interesting one for a heavy WIMP scenario 4], are poorly tested in literature; on the other hand, some studies are performed for very heavy DM with $m_{\mathrm{DM}}>10 \mathrm{TeV}$, which is disfavored by recent astrophysical observations [4]. In addition, the greater the mass, the greater the uncertainty of the annihilation scheme and the greater the degrees of freedom to tune the 
annihilation chain and fit the data.

It must be stressed that all recent observations point toward a $\mathrm{TeV}$-ish paradigm and suggest to look above $1 \mathrm{TeV}$ for WIMP DM masses [4; at the same time too high DM masses $\geq 10 \mathrm{TeV}$ could introduce some conflicts and could be not too suitable to fit the AMS-02 2014 positron fraction and its supposed flattening, due to the achievement of a maximum of the positron production. It is more advisable to avoid the $t, h, W^{ \pm}$annihilation scenarios associated to very high masses and too high annihilation cross sections 21].

The previous fits have one order of magnitude of span in the annihilation cross section; the ensemble of the fits prescriptions could be approximately described as a rectangle in the $\langle\sigma \nu\rangle-m_{\text {DM }}$ plane: $\left(5 \times 10^{-(24 \div 23)} \mathrm{cm}^{3} \mathrm{~s}^{-1}\right) \times(1 \div$ $4 \mathrm{TeV})$. This must be translated in the $f_{\mathrm{eff}}\langle\sigma \nu\rangle-m_{\mathrm{DM}}$ plane: if we take $0.1 \leq f_{\text {eff }} \leq 1$, the most general permitted window becomes $\left(5 \times 10^{-(25 \div 23)} \mathrm{cm}^{3} \mathrm{~s}^{-1}\right) \times(1 \div 4 \mathrm{TeV})$. But such a rectangle is very loose and it is based on standard assumptions which can be easily extended. In fact the constraints from AMS-02 positron data greatly relax introducing a Dark Disk (DD) in addition to a dark halo: this ensures dynamical enhancements of the DM annihilation cross section within the high density DD, $\rho_{\mathrm{DDDM}} \gg$ $0.4 \mathrm{GeV} \mathrm{cm} \mathrm{cm}^{-3}$, without the need of $\langle\sigma \nu\rangle>10^{-24} \mathrm{~cm}^{3} \mathrm{~s}^{-1}$, 28. For indirect detection, the DD scenario could easily accommodate a large boost factor from local density enhancement in the range $10 \div 1000$, depending on the disk height.

In addition, the constraints become less stringent if one consider both DM and pulsars as sources of primary positrons 29]. Pulsars and DD hypothesis can only relax the DM bounds, enlarging the allowed region in the $f_{\text {eff }}\langle\sigma \nu\rangle-m_{\text {DM }}$ plane.

For what concerns the uncertainties which afflict CR propagation physics, we still do not have a complete and wellposed understanding of the CR lepton problematic: nonstandard propagation models may be introduced to account for part or all of the positrons measured in space 30]). Hence the constraints obtained from the positron sector (positron spectrum and positron fraction) strongly depend on the underlying CR propagation model and certainly on the AMS-02 measurements errors. Thanks to AMS-02 unprecedented precision it will be soon possible to fix an almost univocal scheme of propagation of cosmic rays in our galaxy, allowing to correctly compute the effective background for DM indirect searches: in fact, tiny variations of the most significant parameters, such as the diffusive halo thickness, the diffusion coefficient and exponent and the electrons spectral indices, may lead to misleading interpretations of AMS-02 data, pointing to an incorrect scenario. In Fig. 5 a qualitative illustration of propagation and measurement uncertainties, performed with PPPC4DMID [31, show how unstable these fits are: a best-fit point in the $\langle\sigma \nu\rangle-m_{\mathrm{DM}}$ plain carries up to $50 \%$ uncertainty in the choice of the annihilation cross section and DM mass values.

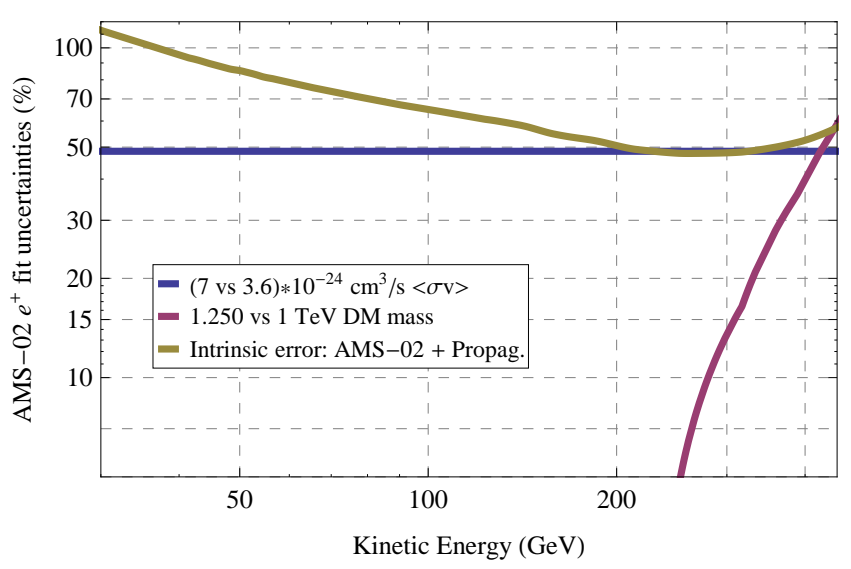

Figure 5: Uncertainties comparison associated to cosmic positron spectrum measured by AMS-02. We plot the ratio between a reference positron fit with $\langle\sigma \nu\rangle=7 \times 10^{-24} \mathrm{~cm}^{3} \mathrm{~s}^{-1}$ and a set with about half the annihilation cross section (blue curve) and the ratio between a reference positron fit with $m_{\mathrm{DM}}=1 \mathrm{TeV}$ and one with $m_{\mathrm{DM}}=1.25 \mathrm{TeV}$ (purple curve). As long the two curves lie below the dark yellow line, which represent the combination of the two main uncertainties sources, from CR propagation (the min-max sets span in 31]) and from AMS-02 errors, the best-fit point can be adjusted in the $\langle\sigma \nu\rangle-m_{\mathrm{DM}}$ plain.

From Fig. 5 one argues that DM fits to AMS-02 positrons and positron fraction with standard propagation models, up to $(400 \div 450) \mathrm{GeV}$, suffer large uncertainties, of the order of $(25 \div 50) \%$, if taken individually. This is the degree of uncertainty from the most precise space experiment which measures CR fluxes: when a fit on PAMELA, Fermi (or AMS-01, HEAT, HESS) data is performed, an uncertainty at least three times the AMS-02 ones should be addressed: it could imply almost one order of magnitude in the annihilation cross section and more than $1 \mathrm{TeV}$ for TeV-ish DM masses.

Finally, when exploring the DM $\langle\sigma \nu\rangle-m_{\mathrm{DM}}$ space, other underlying parameters are fixed, such as the dark matter halo shape and the next-to-leading order (NLO) corrections to primary positrons from DM annihilation. The choice of the DM radial profile is not too significant, whereas full calculations of the NLO and NNLO electroweak (EW) corrections may modify the primary DM fluxes up to one order of magnitude [32, 33, 34, producing more light final states than expected in LO calculations and allowing lower values of the annihilation cross section: they are relevant for spectra predictions especially when $m_{\mathrm{DM}}$ is much larger than the EW scale and EW bremsstrahlung is permitted.

\section{Results and Conclusions}

We consider the collection of fits discussed in Section 3 applying the constraint $0.1<f_{\text {eff }}<0.4$, according to the up-to-date calculations derived in [16, 17], where 0.4 is preferred only for the electron channels for non $\mathrm{TeV}$ ish DM candidates, an overall CR allowed region is ob- 
tained (Fig. 6), which is something like $\left(4 \times 10^{-25} \div 1.5 \times\right.$ $\left.10^{-23} \mathrm{~cm}^{3} \mathrm{~s}^{-1}\right) \times(1 \div 4 \mathrm{TeV})$. The qualitative fraction of this region permitted by CMB observations is about $9 \%$, $4 \%$ and $2 \%$ of the total area obtained combining cosmic rays fits, assuming $f_{\text {eff }}=0.1,0.15,0.2$ respectively.

Our analysis is compatible with the one presented in [14]; in 14 the region allowed by Planck is $\sim 2 \%$.

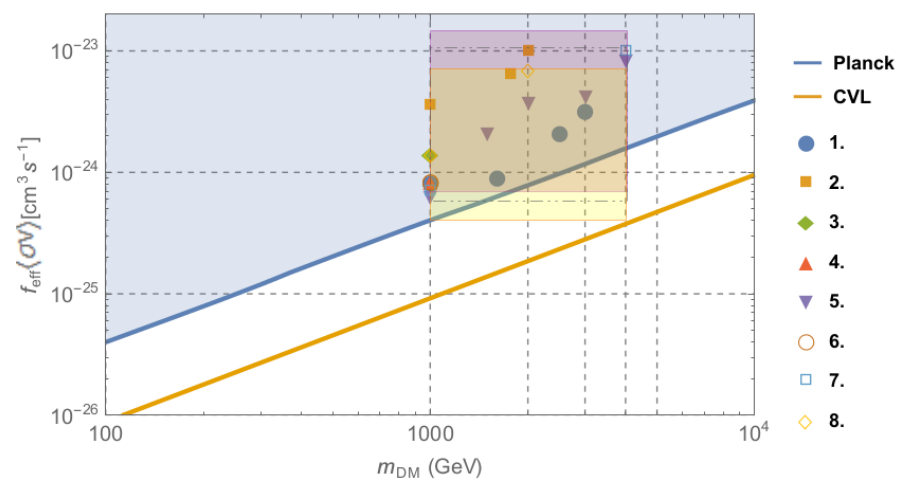

Figure 6: Allowed parameters space for indirect search on $f_{\text {eff }}\langle\sigma \nu\rangle-$ $m_{\text {DM }}$ plane from AMS-02/Fermi/PAMELA (dot-dashed rectangle), along with Planck 2015 bound (blue line). Only a Cosmic Variance Limited (CVL) experiment (black line) could completely falsify the $\mathrm{CR}$ indirect search constraints for TeV-ish candidates. The points corresponding to the best-fits are the ones presented in Section 3 for $f_{\text {eff }}=0.15$. The rectangles for $f_{\text {eff }}=0.1$ (yellow) and $f_{\text {eff }}=0.2$ (purple) are also drawn.

Planck 2015 CMB data appear not to completely exclude annihilating DM as primary source for AMS-02 positron excess, regardless the choice of the recombination efficiency value. From Fig. 6 it emerges that future improvement on CMB measurements 35] could hardly falsify the dark matter interpretation of cosmic-rays antiparticles, for what concerns $\mathrm{TeV}$-ish dark matter candidates. In addition, fundamental uncertainties from CR propagation physics and from DD hypothesis, pulsars contributions and alternative $\mathrm{CR}$ propagation models, not reported in Fig. 6. could almost arbitrarily enlarges the window in the $f_{\text {eff }}\langle\sigma \nu\rangle-m_{\text {DM }}$ plane, up to $10 \mathrm{TeV}$ particles and down to nearly thermal cross sections. Incoming CR high statistics measurements of leptons and nuclei from AMS-02 will allow us to deepen the examination of this important issue, narrowing CR uncertainties and DM properties. Besides that, the information from the antiproton channel is mandatory to put more consistent and coherent constraints on the annihilation capability of the DM candidate, because CR antiprotons have less important backgrounds [5, so granting more precise estimations. A cross check based on a hadron-lepton channels comparison will improve our understanding of DM annihilation and probably enlarge the analysis up to the $10 \mathrm{TeV}$ scale.

\section{Acknoledgements}

We wish to thank Fabio Finelli and Daniela Paoletti for useful comments and suggestions on the draft. MB acknowledge support by the "ASI/INAF Agreement 2014024-R.0 for the Planck LFI Activity of Phase E2".

\section{References}

\section{References}

[1] L. Accardo et al. [AMS Collaboration], Phys. Rev. Lett. 113, 121101 (2014).

[2] M. Aguilar et al. [AMS Collaboration], Phys. Rev. Lett. 113, 221102 (2014)

[3] M. Aguilar et al. [AMS Collaboration], Phys. Rev. Lett. 113, 121102 (2014).

[4] N. Masi, Eur. Phys. J. Plus 130, no. 4, 69 (2015).

[5] N. Masi, Lambert Academic Publishing/Verlag, Berlin, 2013, ISBN 978-3-659-46138-5.

[6] M. Cirelli, M. Kadastik, M. Raidal and A. Strumia, Nucl. Phys. B 813, 1 (2009) [Nucl. Phys. B 873, 530 (2013)] arXiv:0809.2409 [hep-ph]].

[7] T. R. Slatyer, N. Padmanabhan and D. P. Finkbeiner, Phys. Rev. D 80, 043526 (2009) arXiv:0906.1197 [astro-ph.CO]].

[8] N. Arkani-Hamed, D. P. Finkbeiner, T. R. Slatyer and N. Weiner, Phys. Rev. D 79, 015014 (2009) arXiv:0810.0713 [hep-ph]].

[9] X. L. Chen and M. Kamionkowski, Phys. Rev. D 70, 043502 (2004) astro-ph/0310473.

[10] N. Padmanabhan and D. P. Finkbeiner, Phys. Rev. D 72, 023508 (2005) astro-ph/0503486.

[11] S. Galli, F. Iocco, G. Bertone and A. Melchiorri, Phys. Rev. D 84, 027302 (2011) arXiv:1106.1528 [astro-ph.CO]].

[12] D. P. Finkbeiner, S. Galli, T. Lin and T. R. Slatyer, Phys. Rev. D 85, 043522 (2012) arXiv:1109.6322 [astro-ph.CO]].

[13] S. Galli, F. Iocco, G. Bertone and A. Melchiorri, Phys. Rev. D 80, 023505 (2009) arXiv:0905.0003 [astro-ph.CO]].

[14] P. A. R. Ade et al. [Planck Collaboration], arXiv:1502.01589 [astro-ph.CO].

[15] I. Cholis and D. Hooper, Phys. Rev. D 88, 023013 (2013) arXiv:1304.1840 [astro-ph.HE]].

[16] T. R. Slatyer, arXiv:1506.03811 [hep-ph].

[17] T. R. Slatyer, arXiv:1506.03812 [astro-ph.CO].

[18] M. Aguilar et al. [AMS Collaboration], Phys. Rev. Lett. 110, 141102 (2013).

[19] M. Ackermann et al. [Fermi-LAT Collaboration], Phys. Rev. D 82, 092004 (2010) arXiv:1008.3999 [astro-ph.HE]].

[20] O. Adriani et al. [PAMELA Collaboration], Phys. Rev. Lett. 106, 201101 (2011) arXiv:1103.2880 [astro-ph.HE]].

[21] M. Boudaud, S. Aupetit, S. Caroff, A. Putze, G. Belanger, Y. Genolini, C. Goy and V. Poireau et al., Astron. Astrophys. 575, A67 (2015) arXiv:1410.3799 [astro-ph.HE]].

[22] [Fermi-LAT Collaboration], arXiv:1501.02003 [astro-ph.HE].

[23] L. Feng, R. Z. Yang, H. N. He, T. K. Dong, Y. Z. Fan and J. Chang, Phys. Lett. B 728, 250 (2014) arXiv:1303.0530 [astroph.HE]].

[24] Z. P. Liu, Y. L. Wu and Y. F. Zhou, Phys. Rev. D 88, 096008 (2013) arXiv:1305.5438 [hep-ph]].

[25] A. Ibarra, A. S. Lamperstorfer and J. Silk, Phys. Rev. D 89, no. 6, 063539 (2014) arXiv:1309.2570 [hep-ph]].

[26] S. J. Lin, Q. Yuan and X. J. Bi, Phys. Rev. D 91, no. 6, 063508 (2015) arXiv:1409.6248 [astro-ph.HE]].

[27] A. Lopez, C. Savage, D. Spolyar and D. Q. Adams, arXiv:1501.01618 [astro-ph.CO].

[28] J. Fan, A. Katz, L. Randall and M. Reece, Phys. Dark Univ. 2, 139 (2013) arXiv:1303.1521 [astro-ph.CO]].

[29] J. Feng and H. H. Zhang, arXiv:1504.03312 [hep-ph]].

[30] P. Mertsch and S. Sarkar, Phys. Rev. D 90, 061301 (2014) arXiv:1402.0855 [astro-ph.HE]].

[31] M. Cirelli, G. Corcella, A. Hektor, G. Hutsi, M. Kadastik, P. Panci, M. Raidal and F. Sala et al., JCAP 1103, 051 (2011) [JCAP 1210, E01 (2012)] arXiv:1012.4515 [hep-ph]].

[32] P. Ciafaloni, D. Comelli, A. De Simone, A. Riotto and A. Urbano, JCAP 1206, 016 (2012) arXiv:1202.0692 [hep-ph]]. 
[33] A. De Simone, J. Phys. Conf. Ser. 375, 012046 (2012) arXiv:1201.1443 [hep-ph]].

[34] A. Hryczuk and R. Iengo, JHEP 1201, 163 (2012) [JHEP 1206, 137 (2012)] arXiv:1111.2916 [hep-ph]].

[35] M. S. Madhavacheril, N. Sehgal and T. R. Slatyer, Phys. Rev. D 89, 103508 (2014) arXiv:1310.3815 [astro-ph.CO]]. 